\title{
Secretome characterization of clinical isolates from the Mycobacterium abscessus complex provides insight into antigenic differences
}

Fernanda Cornejo-Granados ${ }^{1}$, Thomas A. Kohl ${ }^{2,3}$, Flor Vásquez Sotomayor ${ }^{4}$, Sönke Andres ${ }^{4}$, Rogelio Hernández-Pando ${ }^{5}$, Juan Manuel Hurtado-Ramirez ${ }^{1}$, Christian Utpatel ${ }^{2,3}$, Stefan Niemann ${ }^{2,3}$, Florian P. Maurer ${ }^{3,4,6^{*}+}$ and Adrian Ochoa-Leyva ${ }^{1 *+}$

\begin{abstract}
Background: Mycobacterium abscessus (MAB) is a widely disseminated pathogenic non-tuberculous mycobacterium (NTM). Like with the M. tuberculosis complex (MTBC), excreted / secreted (ES) proteins play an essential role for its virulence and survival inside the host. Here, we used a robust bioinformatics pipeline to predict the secretome of the M. abscessus ATCC 19977 reference strain and 15 clinical isolates belonging to all three MAB subspecies, M. abscessus subsp. abscessus, M. abscessus subsp. bolletii, and M. abscessus subsp. massiliense.

Results: We found that $\sim 18 \%$ of the proteins encoded in the MAB genomes were predicted as secreted and that the three MAB subspecies shared $>85 \%$ of the predicted secretomes. MAB isolates with a rough (R) colony morphotype showed larger predicted secretomes than isolates with a smooth (S) morphotype. Additionally, proteins exclusive to the secretomes of MAB R variants had higher antigenic densities than those exclusive to $S$ variants, independent of the subspecies. For all investigated isolates, ES proteins had a significantly higher antigenic density than non-ES proteins. We identified 337 MAB ES proteins with homologues in previously investigated M. tuberculosis secretomes. Among these, 222 have previous experimental support of secretion, and some proteins showed homology with protein drug targets reported in the DrugBank database. The predicted MAB secretomes showed a higher abundance of proteins related to quorum-sensing and Mce domains as compared to MTBC indicating the importance of these pathways for MAB pathogenicity and virulence. Comparison of the predicted secretome of M. abscessus ATCC 19977 with the list of essential genes revealed that 99 secreted proteins corresponded to essential proteins required for in vitro growth.

(Continued on next page)
\end{abstract}

\footnotetext{
* Correspondence: aochoa@ibt.unam.mx; fmaurer@fz-borstel.de

${ }^{\dagger}$ Florian P. Maurer and Adrian Ochoa-Leyva contributed equally to this work.

${ }^{3}$ German Center for Infection Research (DZIF), Partner site

Hamburg-Lübeck-Borstel, Borstel, Germany

'Departamento de Microbiología Molecular, Instituto de Biotecnología, Universidad Nacional Autonoma de México, Cuernavaca, Morelos, Mexico

Full list of author information is available at the end of the article
}

(c) The Author(s). 2021 Open Access This article is licensed under a Creative Commons Attribution 4.0 International License, which permits use, sharing, adaptation, distribution and reproduction in any medium or format, as long as you give appropriate credit to the original author(s) and the source, provide a link to the Creative Commons licence, and indicate if changes were made. The images or other third party material in this article are included in the article's Creative Commons licence, unless indicated otherwise in a credit line to the material. If material is not included in the article's Creative Commons licence and your intended use is not permitted by statutory regulation or exceeds the permitted use, you will need to obtain permission directly from the copyright holder. To view a copy of this licence, visit http://creativecommons.org/licenses/by/4.0/. The Creative Commons Public Domain Dedication waiver (http://creativecommons.org/publicdomain/zero/1.0/) applies to the data made available in this article, unless otherwise stated in a credit line to the data. 


\begin{abstract}
(Continued from previous page)
Conclusions: This study represents the first systematic prediction and in silico characterization of the MAB secretome. Our study demonstrates that bioinformatics strategies can help to broadly explore mycobacterial secretomes including those of clinical isolates and to tailor subsequent, complex and time-consuming experimental approaches accordingly. This approach can support systematic investigation exploring candidate proteins for new vaccines and diagnostic markers to distinguish between colonization and infection. All predicted secretomes were deposited in the Secret-AAR web-server (http://microbiomics.ibt.unam.mx/tools/aar/index.php).
\end{abstract}

Keywords: Bioinformatics, Antigenicity, M. abscessus subspecies, In silico analysis, Vaccinology

\section{Background}

Non-tuberculous mycobacteria (NTM) are widely disseminated, mostly saprophytic and partly opportunistic bacteria. The prevalence of NTM in clinical specimens has increased globally, and in some industrialized countries, infections caused by NTM are becoming more common than tuberculosis (TB). Infections caused by M. abscessus $(\mathrm{MAB})$ are particularly challenging to manage due to the extensive innate resistance of $\mathrm{MAB}$ against a wide spectrum of clinically available antimicrobials [1]. MAB causes mostly pulmonary and occasionally extrapulmonary infections that can affect all organs in the human body [2]. Current treatments for MAB induced pulmonary disease are long, associated with severe side effects and a cure rate below 50\% [3-5]. MAB is comprised of three subspecies, $M$. abscessus subsp. abscessus, M. abscessus subsp. bolletii and $M$. abscessus subsp. massiliense, hereafter referred to as $\mathrm{MAB}_{\mathrm{A}}, \mathrm{MAB}_{\mathrm{B}}$, and $\mathrm{MAB}_{\mathrm{M}}$, respectively [6]. MAB isolates can show smooth $(S)$ and rough $(R)$ colony morphotypes, a trait that relies on the presence (S) or absence (R) of surface-associated glycopeptidolipids (GPLs) and that correlates with the virulence of the strain [7-10]. Transitioning from high-GPL to low-GPL production is observed in sequential MAB isolates obtained from patients with chronic underlying pulmonary disease. In these patients, S-to- $\mathrm{R}$ conversion is thought to present a selective advantage as the aggregative properties of MAB R variants strongly affect intracellular survival. The selective advantage is also related to the loss of immunogenic GPLs. In addition, a propensity to grow as extracellular cords allows these low-GPL producing bacilli to escape innate immune defenses [10].

The complete set of proteins excreted / secreted (ES) by a bacterial cell is referred to as its secretome. The secretome is involved in critical biological processes such as cell adhesion, migration, cell-to-cell communication and signal transduction [11] ES proteins are considered an important source of molecules for serological diagnosis. Also, secreted proteins can be highly antigenic due to their immediate availability to the host immune system and are thus of interest in vaccinology [12, 13]. So far, there have been few efforts to experimentally determine the secretome of $\mathrm{MAB}$, and in particular, the secretomes of clinical MAB isolates [14-17]. Nowadays, sequencing and bioinformatics strategies can be explored for the systematized prediction of ES proteins from bacterial genomes $[18,19]$. Recently, a robust bioinformatics pipeline for predicting and analyzing the complete in silico secretome of two clinical $M$. tuberculosis (MTB) genomes was published showing higher overall agreement with an experimental secretome compiled from literature than two previously reported secretomes for $M$. tuberculosis H37Rv [19].

To gain further insights into MAB ES proteins and their association with virulence and pathogenicity we here sequenced and assembled the genomes of 15 clinical MAB isolates belonging to all three subspecies including $\mathrm{S}$ and $\mathrm{R}$ morphotypes. We then adapted the bioinformatics strategy previously established for MTB to predict and analyze the complete set of ES proteins encoded in these isolates and in the M. abscessus ATCC 19977 type strain, and compared it with our previous findings for MTB [19].

\section{Results}

Genome assembly, secretome prediction and annotation We sequenced the genomes of 15 pulmonary and extrapulmonary (skin, tissue, lymph node, and blood) MAB isolates obtained from patients in Germany comprising all three MAB subspecies (Table 1 and Additional file 1: Table S1). For each genome, we obtained an average of $2,601,444$ quality-filtered reads. After de novo assembly, we obtained from 38 to 78 contigs (mean $=58$ contigs) with genome coverage of 217 - to 368 -fold (mean $=310$ fold) and with an average of 5082 total proteins per genome (Additional file 3: Table S2). Also, we performed a Multilocus Sequence Typing (MLST) analysis at the Pasteur Institute site (https://bigsdb.pasteur.fr/cgi-bin/ bigsdb/bigsdb.pl?db=pubmlst_mycoabscessus_seqdef) to assess the genetic variability among the studied samples. This analysis assigns a Sequence Type (ST) to each strain by looking for sequence variations in seven housekeeping genes and providing information about philogenetic relationship [20]. We observed that eight out of 15 genomes had unique STs, three genomes were not defined and notably, two genomes belonged to ST 117 
Table 1 Clinical isolates metadata and number of ES proteins

\begin{tabular}{|c|c|c|c|c|c|c|c|c|}
\hline Strain & $\begin{array}{l}\text { Accession } \\
\text { number }\end{array}$ & Genome ID & Origin & Phenotype & $\begin{array}{l}\text { Sequence } \\
\text { Type (ST) }\end{array}$ & $\begin{array}{l}\text { Total predicted } \\
\text { proteins }\end{array}$ & $\begin{array}{l}\text { ES } \\
\text { proteins }\end{array}$ & $\begin{array}{l}\% \text { ES } \\
\text { proteins }\end{array}$ \\
\hline \multirow[t]{7}{*}{$\begin{array}{l}\text { M. abscessus subsp. } \\
\text { abscessus }\end{array}$} & $\begin{array}{l}\text { GCA } \\
015499845.1\end{array}$ & 4549-15 & sputum & rough & 1 & 5105 & 929 & 18 \\
\hline & $\begin{array}{l}\text { GCA } \\
015499865.1\end{array}$ & 11351-15 & sputum & rough & 63 & 5138 & 966 & 19 \\
\hline & $\begin{array}{l}\text { GCA } \\
015499835.1\end{array}$ & $8844-15$ & skin & smooth & 246 & 4854 & 956 & 20 \\
\hline & $\begin{array}{l}\text { GCA } \\
015499805.1\end{array}$ & 3563-15 & sputum & smooth & 33 & 5239 & 968 & 18 \\
\hline & $\begin{array}{l}\text { GCA } \\
015499795.1\end{array}$ & 12389-15 & sputum & smooth & 47 & 5276 & 990 & 19 \\
\hline & $\begin{array}{l}\text { GCA } \\
015499765.1\end{array}$ & $2677-16$ & sputum & smooth & 34 & 4900 & 919 & 19 \\
\hline & $\begin{array}{l}\text { GCA } \\
015499745.1\end{array}$ & $2572-17$ & $\begin{array}{l}\text { tissue (breast } \\
\text { implant) }\end{array}$ & NA & $\begin{array}{l}10-46-64-70- \\
261\end{array}$ & 4847 & 874 & 18 \\
\hline \multirow[t]{4}{*}{$\begin{array}{l}\text { M. abscessus subsp. } \\
\text { massiliense }\end{array}$} & $\begin{array}{l}\text { GCA_ } \\
015499715.1\end{array}$ & 14479-15 & sputum & rough & 117 & 5120 & 962 & 19 \\
\hline & $\begin{array}{l}\text { GCA } \\
015499735.1\end{array}$ & 10896-16 & sputum & rough & 117 & 5109 & 950 & 19 \\
\hline & $\begin{array}{l}\text { GCA } \\
015499695.1\end{array}$ & 10003-15 & sputum & smooth & $98-245-271$ & 4835 & 891 & 18 \\
\hline & $\begin{array}{l}\text { GCA } \\
015499655.1\end{array}$ & 16155-15 & sputum & smooth & $98-245-271$ & 4884 & 898 & 18 \\
\hline \multirow[t]{4}{*}{$\begin{array}{l}\text { M. abscessus subsp. } \\
\text { bolletii }\end{array}$} & $\begin{array}{l}\text { GCA } \\
015499665.1\end{array}$ & $11702-16$ & sputum & rough & 161 & 5079 & 931 & 18 \\
\hline & $\begin{array}{l}\text { GCA_ } \\
015499625.1\end{array}$ & 713-16 & lymph node & rough & 52 & 5456 & 1037 & 19 \\
\hline & $\begin{array}{l}\text { GCA } \\
015499615.1\end{array}$ & $7742-15$ & blood culture & smooth & 333 & 4913 & 885 & 18 \\
\hline & $\begin{array}{l}\text { GCA } \\
015499585.1\end{array}$ & 13116-16 & lymph node & smooth & 52 & 5305 & 990 & 19 \\
\hline $\begin{array}{l}\text { M. abscessus subsp. } \\
\text { abscessus }\end{array}$ & CU458896.1 & $\begin{array}{l}\text { reference strain } \\
\text { ATCC19977 }\end{array}$ & - & - & & 4942 & 886 & 18 \\
\hline M. tuberculosis H37Rv & NC_000962.3 & reference strain & - & - & & 4337 & 548 & 13 \\
\hline
\end{tabular}

while other two belonged to ST 52, suggesting they could be highly related (Table 1).

We used a bioinformatics pipeline previously reported by our group [19] to predict the full secretome of all MAB clinical isolates and the widely used reference strain $M$. abscessus ATCC 19977 (GenBank CU458896.1) (Additional file 2: Fig. S1). We obtained an average of 939 ES proteins per genome, representing $\sim 18 \%$ of the total proteome (Table 1). The predicted secretome for the MAB reference strain consisted of 886 proteins. All these proteins showed a BLASTP hit against the NR database but only 494 (55.8\%) could be annotated with GO terms.

We analyzed the over-representation of GO terms in the secretome of M. abscessus ATCC 19977 as compared to the whole genome. The most significantly enriched GO-terms were: "lytic vacuole" ( $p=9.37 \mathrm{E}-04)$, and "fungal-type vacuole" ( $p=0.004)$ in Cellular Component (Fig. 1a), "serine-type carboxypeptidase" ( $p=1.83 \mathrm{E}-$
04), and "serine-type D-Ala-D-Ala carboxypeptidase" $(p=1.83 \mathrm{E}-04)$ activities in Molecular Function (Fig. 1b) and, "response to inorganic substance" ( $p=5.68 \mathrm{E}-04)$ and "cellular response to oxygen radical" $(p=0.001)$ in the Biological Process category (Fig. 1c). The KEGG pathway mapping of the ES proteins showed that 214 proteins (24.2\%) could be assigned to 100 different KEGG pathways (Table 2), with the ABC transporter pathway being the most abundant $(n=13,1.47 \%)$. Additionally, serine-type D-Ala-D-Ala carboxypeptidases $(p=1.83 \mathrm{E}-04)$ and peptidases $(p=8.40 \mathrm{E}-04)$ were the most significantly abundant enzymes according to the Enzyme Commission (EC) Classes (Additional file 6: Fig. S2), while the Mce/MiaD and PknH-like extracellular domains were the most enriched protein domains (Table 3). Of note, comparably few sequences were assigned to the PE/PPE category $(n=3)$. Notably, after comparing the predicted secretome of $M$. abscessus 
a) Cellular Component

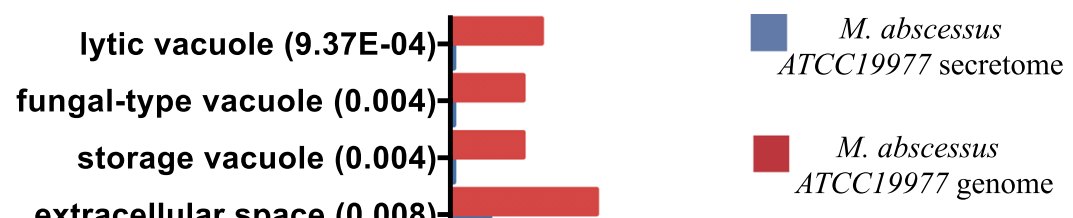

b) Molecular Function

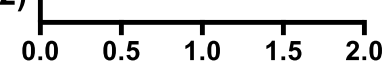

fungal-type vacuole membrane $(0.020)$ -

cytosolic ribosome (0.023)-

endosome membrane $(0.032)$

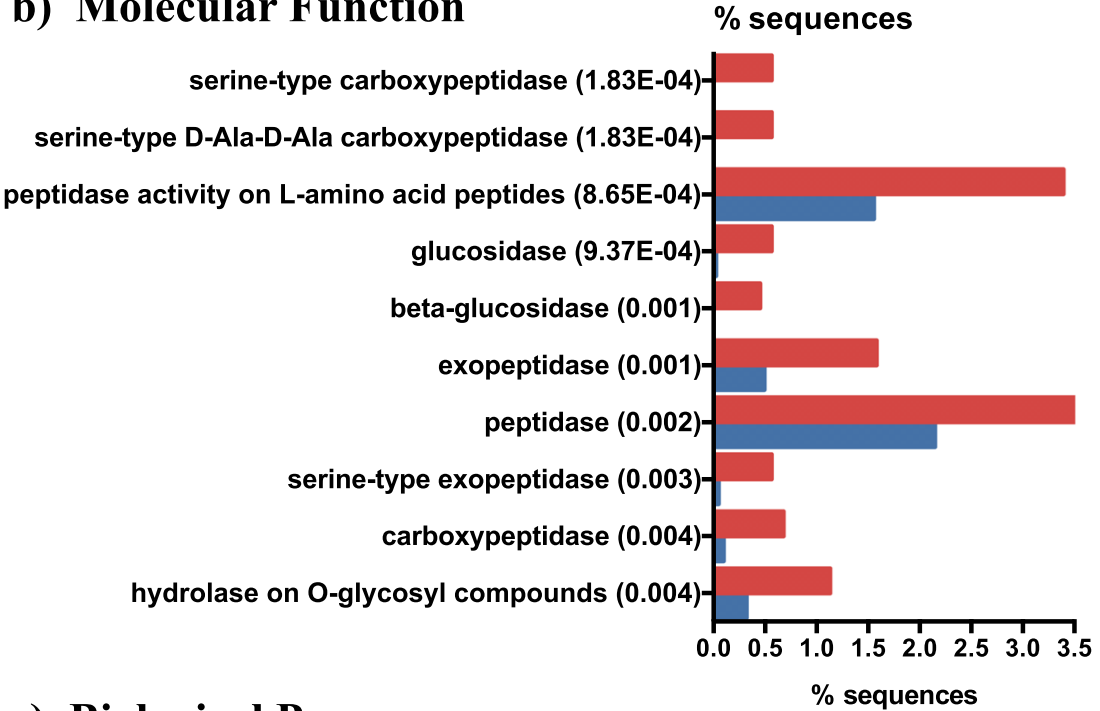

c) Biological Process

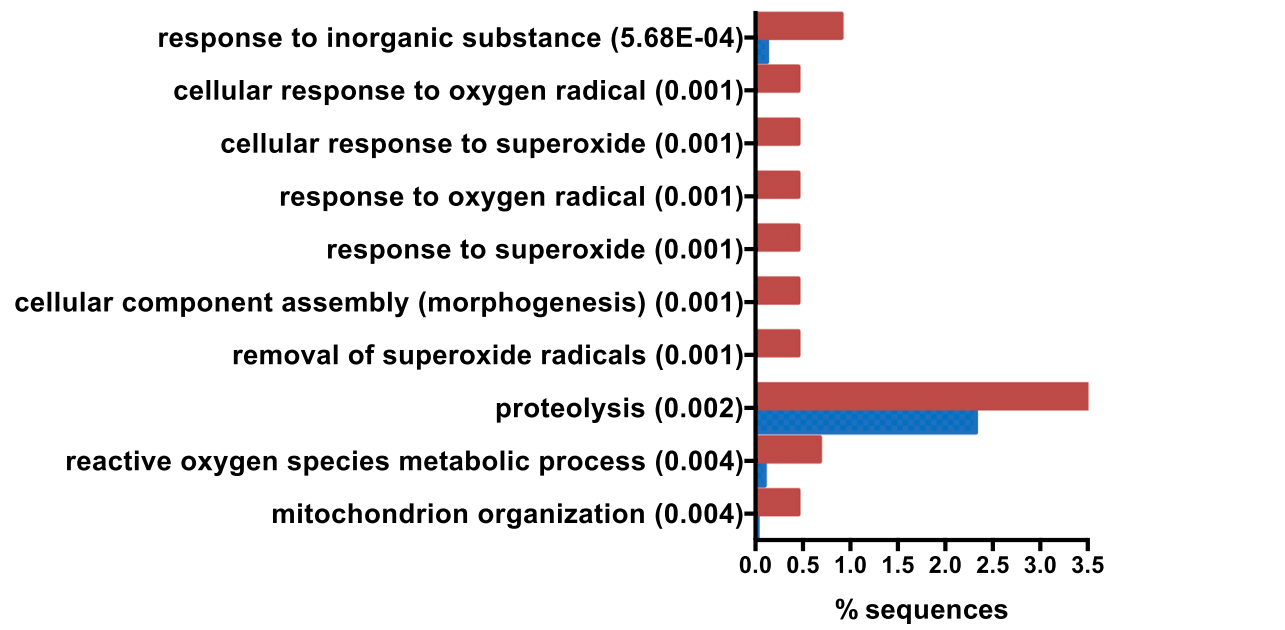

Fig. 1 GO enrichment analysis for the M. abscessus ATCC 19977 reference strain. Top 10 most enriched GO terms for the M. abscessus ATCC 19977 secretome (blue) and complete genome (red) in three categories: a Cellular Component, b Molecular Function and c Biological Process 
Table 2 Top 10 KEGG pathways assigned for M. abscessus ATCC19977 ES proteins

\begin{tabular}{lll}
\hline Ranking & Pathway name & Number of represented ES proteins (\%) \\
\hline 1 & ABC transporters & $13(1.47)$ \\
2 & Two-component system & $9(1.02)$ \\
3 & Quorum sensing & $6(0.68)$ \\
4 & Oxidative phosphorylation & $4(0.45)$ \\
5 & Sulfur metabolism & $4(0.45)$ \\
6 & Glycerolipid metabolism & $4(0.45)$ \\
7 & Peptidoglycan biosynthesis & $4(0.45)$ \\
8 & Protein export & $4(0.45)$ \\
9 & Starch and sucrose metabolism & $3(0.34)$ \\
10 & Glyoxylate and dicarboxylate metabolism & $3(0.34)$ \\
\hline
\end{tabular}

ATCC 19977 with a list of essential genes published by Laencina et al. [17], we found that 99 (11.17\%) of the predicted ES proteins, corresponded to essential proteins required for in vitro growth.

\section{Comparison of $M$. abscessus subspecies core secretomes}

We analyzed the differences between the predicted secretomes of the three MAB subspecies. To this end, we defined the core secretome of each subspecies as the set of proteins shared between all secretomes of isolates belonging to $\mathrm{MAB}_{\mathrm{A}}, \mathrm{MAB}_{\mathrm{B}}$, and $\mathrm{MAB}_{\mathrm{M}}$, respectively. The resulting core secretomes contained $735\left(\mathrm{MAB}_{\mathrm{A}}\right)$, $794\left(\mathrm{MAB}_{\mathrm{B}}\right)$, and $813\left(\mathrm{MAB}_{\mathrm{M}}\right)$ proteins (Fig. 2a).

Given that our study considered a limited number of de novo assembled genomes, we additionally compared the predicted core secretomes to 60 additional MAB genomes available in NCBI (Additional file 4: Table S3). We found that an average of 99.78, 99.12, and 98.59\% of our core secretomes was also present in the additional $M A B_{A}, M A B_{B}$, and $M A B_{M}$ genomes, respectively, further corroborating the validity of the predicted subspecies core secretomes for other MAB isolates.

We then determined the respective Abundance of Antigenic Regions (AAR) values to estimate antigenic densities for the protein sets in each core secretome. The average $A A R$ values from most to least antigenic were: 40.24 for $\mathrm{MAB}_{\mathrm{A}}, 40.75$ for $\mathrm{MAB}_{\mathrm{B}}$, and 41.38 for $\mathrm{MAB}_{\mathrm{M}}$ with no statistically significant difference between them.

Next, we identified the ES proteins shared between the $M A B_{A}, M A B_{B}$, and $M A B_{M}$ core secretomes. We found that 704 proteins $(86.5 \%)$ were shared among $M A B_{A}$, $M A B_{B}$, and $\mathrm{MAB}_{\mathrm{M}}$ with an AAR value of 41.17 (Fig. 2b). The AAR values for the protein sets exclusively found in the $M A B_{A}, M A B_{B}$, or $M_{A B}$ secretome were 33.58, 41.22, and 43.13, respectively, with the $M_{A B}$ dataset showing a significantly lower AAR value indicating higher antigenicity than the others ( $p<0.1$; Fig. $2 \mathrm{~b})$.

\section{Differences in core secretomes between $\mathrm{R}$ and $\mathrm{S}$ morphotypes}

As MAB isolates with $\mathrm{R}$ and $\mathrm{S}$ morphotypes show differences in virulence and pathogenicity, we compared the predicted core secretomes of $\mathrm{R}$ and $\mathrm{S}$ isolates (Fig. 3). We observed that the core secretomes of $\mathrm{R}$ variants were larger $\left(840,924\right.$ and 845 proteins for $\mathrm{MAB}_{\mathrm{A}}, \mathrm{MAB}_{\mathrm{M}}$, and $M A B_{B}$ ) than those of the investigated $S$ variants (764, 872 and 833 proteins, respectively) with no significant differences in antigenic densities as per mean AAR

Table 3 Top 10 most represented protein domains in M. abscessus ATCC19977 secretome

\begin{tabular}{lll}
\hline InterProcode & InterPro description & Number of ES proteins (\%) \\
\hline IPR003399 & Mce/MlaD & $19(2.14)$ \\
IPR026954 & PknH-like extracellular domain & $15(1.69)$ \\
IPR032407 & Haemophore, haem-binding & $10(1.13)$ \\
IPR020846 & Major facilitator superfamily domain & $7(0.79)$ \\
IPR013766 & Thioredoxin domain & $6(0.68)$ \\
IPR000064 & Endopeptidase, NLPC/P60 domain & $6(0.68)$ \\
IPR001638 & Solute-binding protein family 3/N-terminal domain of MItF & $6(0.68)$ \\
IPR000675 & Cutinase/acetylxylan esterase & $6(0.68)$ \\
IPR005490 & L,D-transpeptidase catalytic domain & $5(0.56)$ \\
IPR000073 & Alpha/beta hydrolase fold-1 & $5(0.56)$
\end{tabular}




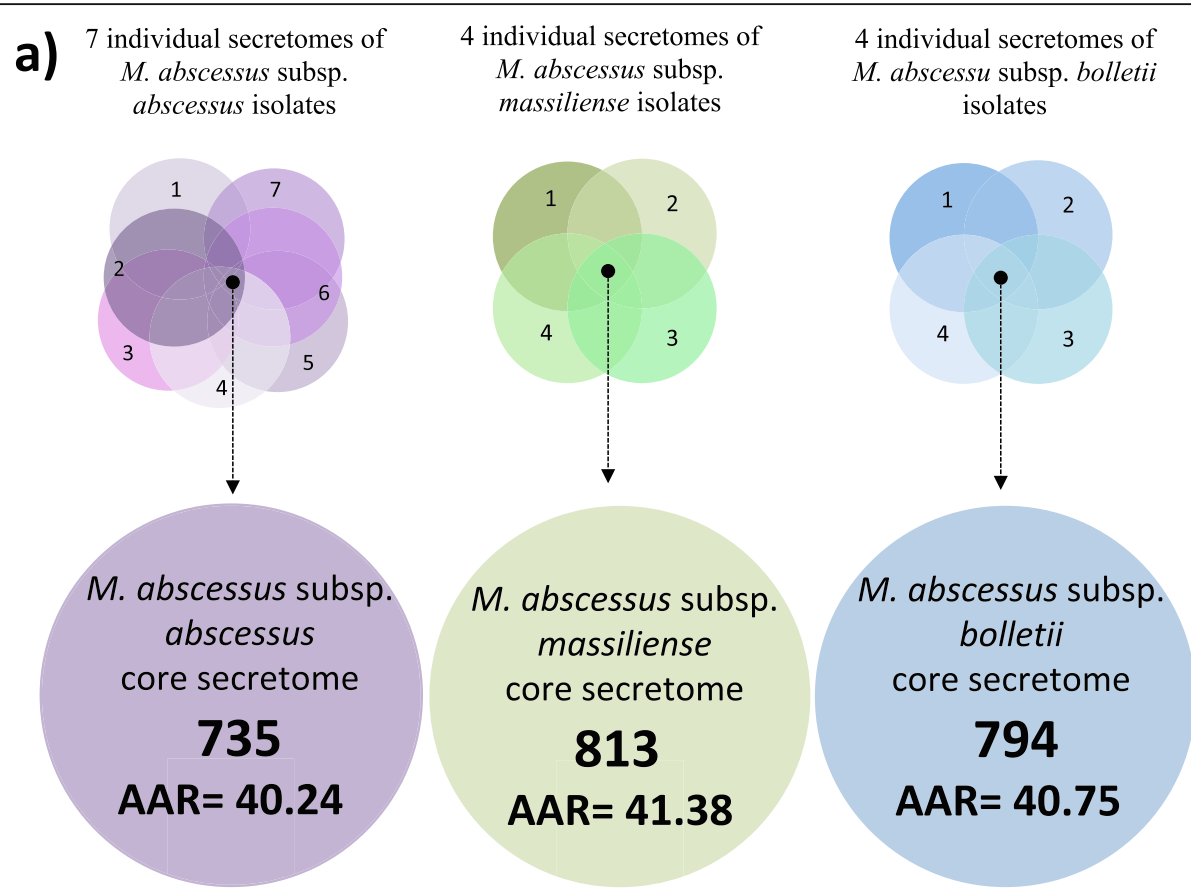

b)

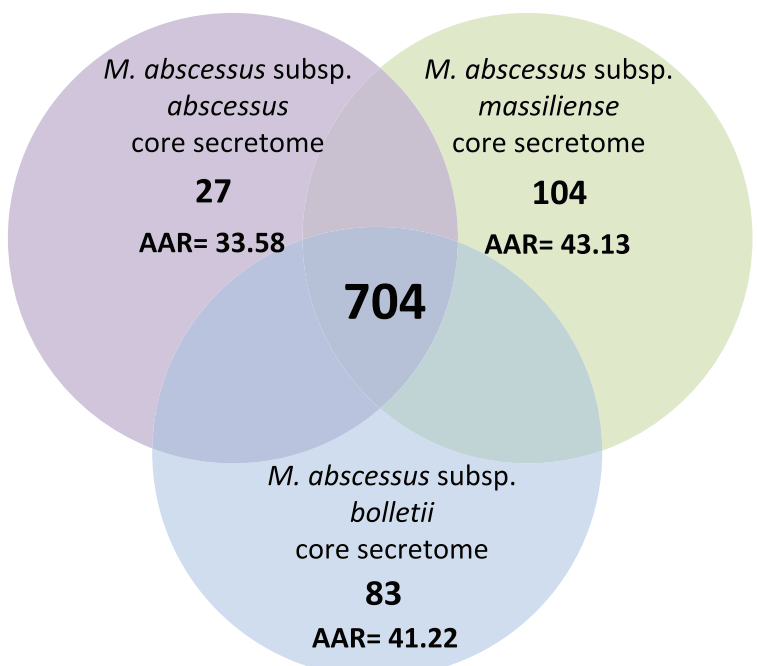

Fig. 2 Venn diagram between the core secretomes of the three M. abscessus subspecies. a Number of total proteins contained in the core secretome of each subspecies. b Shared and unique proteins between the three subspecies as per BLASTP (E-value 1.0E-3)

value (Fig. 3). Intra-subspecies comparison of $\mathrm{S}$ and $\mathrm{R}$ secretomes revealed that $96.4,90.7$ and $95 \%$ of the identified ES proteins were found in both $\mathrm{R}$ and $\mathrm{S}$ morphotypes for $M A B_{A}, M A B_{M}$ and $M A B_{B}$ respectively. The number of unique proteins was larger in the core secretome of the $\mathrm{R}$ morphotypes $(n=93,109$, and 48 for $\mathrm{MAB}_{\mathrm{A}}, \mathrm{MAB}_{\mathrm{M}}$, and $\mathrm{MAB}_{\mathrm{B}}$ ) as compared to the $\mathrm{S}$ morphotypes ( $n=9,76$, and 35 , respectively; Fig. 3 ).

Interestingly, antigenic densities for the unique ES proteins of the $\mathrm{R}$ morphotypes were higher ( $\mathrm{AAR}=$ 40.84, 36.71, and 35.59 for $\mathrm{MAB}_{\mathrm{A}}, \mathrm{MAB}_{\mathrm{M}}$, and $M \mathrm{MB}_{\mathrm{B}}$ ) than for the proteins exclusive to the $\mathrm{S}$ morphotypes irrespective of the subspecies (AAR $=45.43,37.72$, and 42.14; Fig. 3). To assess if the AAR values of these specific protein sets were different from same-sized protein sets randomly chosen from the respective core secretomes, we created 1000 random sets of $109,93,76,48$, 35 and 9 proteins and calculated the AAR value for each set. Then, we determined an empirical $p$-value based on the number of random protein sets that equaled or exceeded the AAR value for each protein dataset as was previously suggested by Cornejo-Granados et al. [19]. 


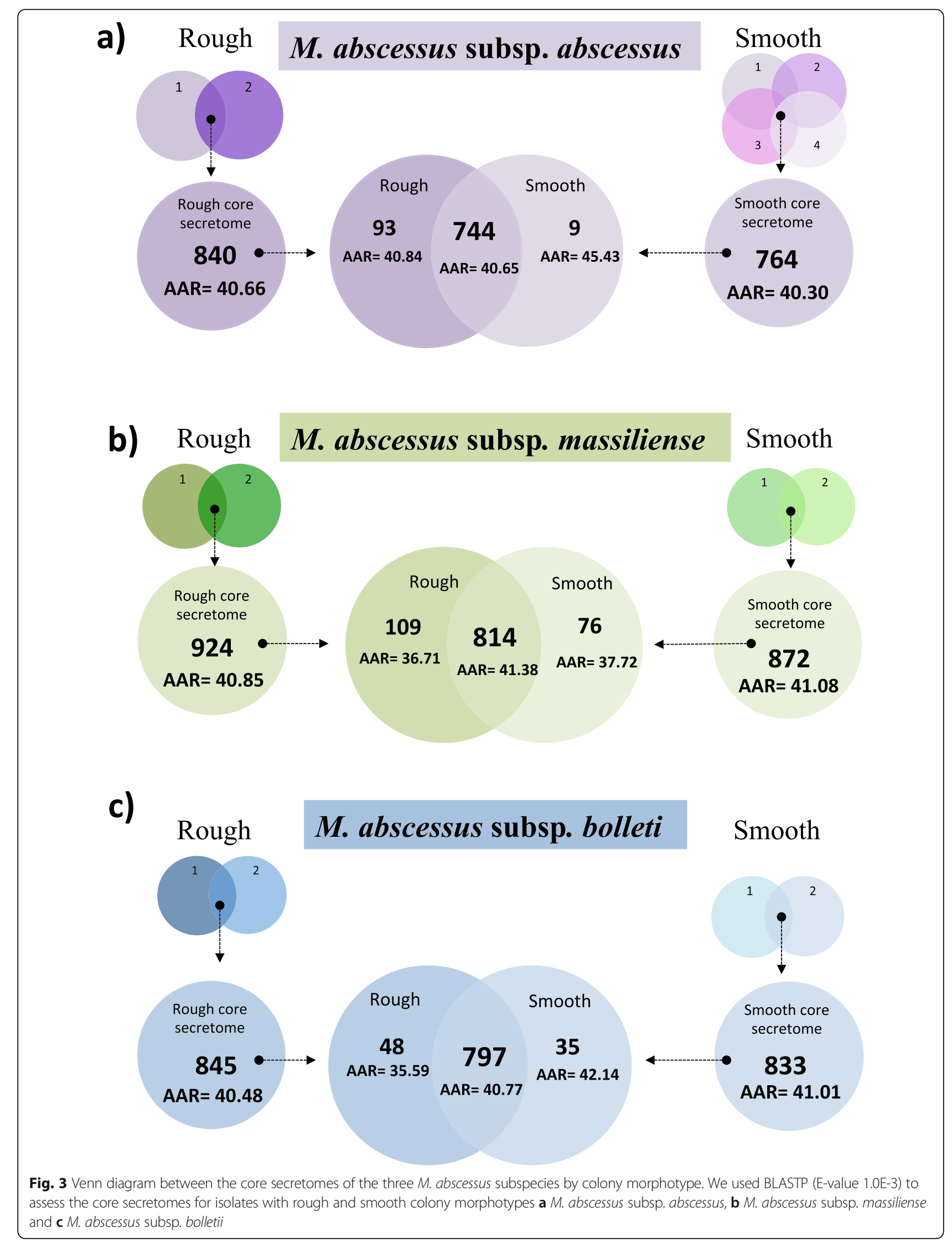


We found that the ES proteins exclusive to the R morphotypes of $\mathrm{MAB}_{\mathrm{M}}$ and $\mathrm{MAB}_{\mathrm{B}}$ had significantly $(p<$ $0.05)$ higher antigenic densities than randomly constructed protein sets (Additional file 5: Table S4).

Finally, we determined the MAB core secretomes by sample origin (pulmonary, extrapulmonary, blood). This resulted in 706 ES proteins shared among the ten pulmonary isolates, 758 proteins shared among the four extrapulmonary isolates, and 885 proteins for the single isolate grown from a blood sample. However, as per the GO, KEGG, and antigenicity analyses, we did not find any distinct characteristics specific to either sample source and, hence, type of infection.

\section{Antigenicity of ES and non-ES proteins}

It has previously been reported for different microorganisms including MTB that ES proteins tend to be more antigenic than non-ES proteins $[18,19,21]$. We thus tested if this was also true for the investigated MAB isolates. First, we found that the antigenic densities as indicated by mean AAR values were similar among all isolates irrespective of subspecies or morphotype within the same cell compartment, i.e. for ES, non-ES, intracellular (incell) and transmembrane (TM) proteins (Fig. 4). Second, we found that antigenic densities were significantly higher in ES proteins as compared to non-ES proteins in all isolates (AAR $=40.57$ and 43.60, respectively; $p$-value <0.0001) (Fig. 4). However, within the non-ES category, incell proteins showed even higher antigenic densities (AAR $=39.04)$ than the predicted ES proteins $(p<0.0001)$ while the lowest overall antigenic densities were observed for the TM category (AAR $=59.23$; $p<$ 0.0001).

\section{Comparison of M. abscessus and M. tuberculosis secretomes}

Lastly, we compared the predicted secretome of $M$. abscessus ATCC 19977 against the previously reported secretome of M. tuberculosis H37Rv [19]. We observed that the $M$. abscessus secretome was predicted to be almost equally antigenic (AAR $=39.63)$ than the $M$. tuberculosis secretome (AAR $=40.37$ ) (Fig. 5). We found 337 MAB ES proteins (38.04\%) with homology to proteins in the predicted MTB secretome (Fig. 5). Interestingly, 222 of these proteins had sequence homology with proteins experimentally reported as secreted in MTB (comparable experimental secretome data for MAB was not available to us) [19] (Additional file 7: Table S5). Furthermore, we determined the average AAR value of the $680 \mathrm{ES}$ proteins shared among the $15 \mathrm{MAB}$ isolates $(\mathrm{AAR}=41.53)$. This value means that antigenic density was lower than for the predicted secretome of $M$. tuberculosis $\mathrm{H} 37 \mathrm{Rv}$ (AAR = 40.37) and two clinical $M$. tuberculosis isolates belonging to the Beijing lineage (isolate C3 AAR $=37.52$ and isolate C4 AAR = 37.55) (Table 4) [19]. Finally, we identified 13 ES proteins with homologues in both MAB and M. tuberculosis, which are listed as targets for various FDA approved drugs (Additional file 8: Table S6).

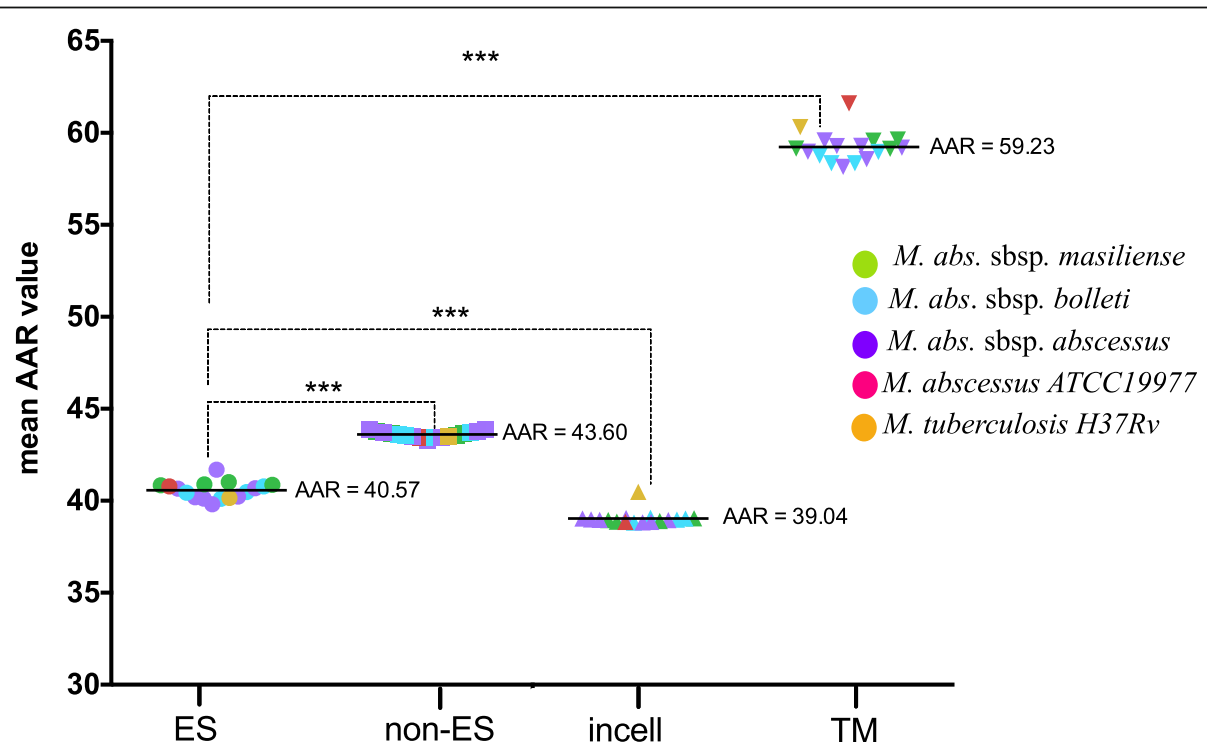

Fig. 4 Comparison between AAR values for Excreted/Secreted (ES), non Excreted/Secreted (non-ES), intracellular (incell) and transmembrane (TM) proteins. AAR values were calculated for each of the 15 genomes sequenced. The $X$-axis shows the cellular compartment and the $Y$-axis shows AAR values for the genomes of each subspecies: M. abscessus subsp. abscessus (green), M. abscessus subsp. bolletii (blue), M. abscessus subsp. massiliense (purple), M. abscessus ATCC19977 (red) and M. tuberculosis H37Rv (orange). Mann-Whitney tests were performed to compare the AAR of each group with a confidence level of $99 \%(* * *, p<0.001)$ 


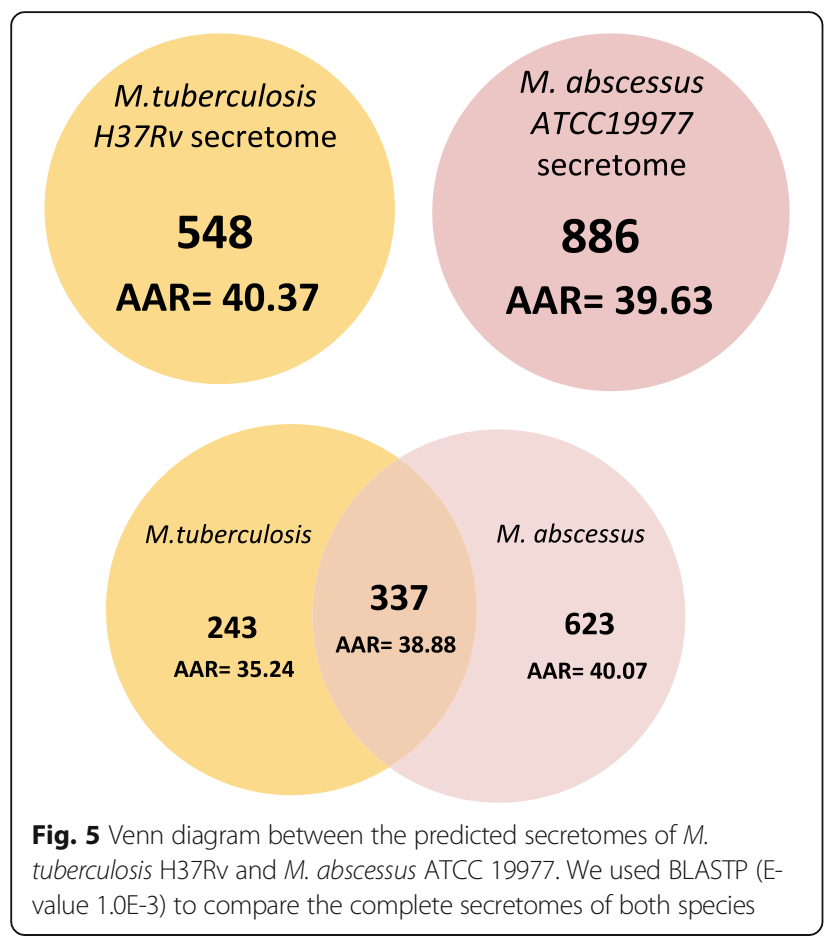

\section{Discussion}

This is the first study that proposes a method for prediction of MAB secretomes based on 15 clinical MAB isolates and the $M$. abscessus ATCC 19977 reference strain. Our results show that an average of $18 \%$ (939 proteins) of the total proteins encoded in the MAB core genome carry sequence patterns indicative of secretion. Notably, this percentage is $6 \%$ greater than the proportion previously reported for several MTB isolates ( 12\%) [19]. Nearly 200 species of mycobacteria have been identified with diverse lifestyles and a high degree of morphological, biochemical, and physiological diversity and a comparative genome analysis suggests that only a relatively small number of genes (1080) are shared between several Mycobacterium species [22, 23]. Moreover, loss of ancestral genes is a well described phenomenon in slowly growing mycobacteria such as MTB and, in particular, M. leprae [24]. In contrast, rapidly growing NTM such as MAB are considered to represent a more ancient evolutionary state, with larger genomes than those of MTB [23, 24]. Thus, it is not surprising that we found a larger number of ES proteins in MAB than MTB. Furthermore, the increased abundance of ES proteins in MAB as compared to MTB could be related to the ability of MAB to cause a different spectrum of disease and to adapt to different environmental settings requiring frequent interaction with a wide variety of host cells and organisms competing for the same ecological niche, likely involving cross species exchange of genetic information, for example by plasmid transfer [25-27]. A similar hypothesis has been suggested for fungal secretomes [28].

The GO and KEGG pathway annotations of the secretomes of M. abscessus ATCC 19977 and the MAB clinical isolates showed enrichment consistent with the characterization of previously reported mycobacterial secretomes $[18,19]$. Interestingly and in line with the increased secretome size as compared to MTB, the KEGG pathway analysis showed a high abundance of the Quorum sensing pathway for the predicted MAB secretomes, which was not present in our previous MTB secretome pathway analysis [19]. The presence of a Quorum sensing pathway would be another similarity shared between MAB and non-mycobacterial pathogens commonly affecting patients with chronic lung disease such as Pseudomonas aeruginosa [29]. In addition, it could be related to the ability of MAB to form biofilms [30, 31], further contributing to the capacity of MAB to tolerate antibiotics and to persist over long periods in the environment [32-35].

The InterPro annotation showed that Mce domains were the most abundant (2.14\%) domains in the MAB reference secretome, while PPE and PE-PGRS domains only corresponded to $0.3 \%$ of the ES protein sequences. This tendency is contrary to our observations for MTB [19], where the PPE and PE-PGRS domains accounted for $\sim 12 \%$ of the secreted proteins and the Mce domains for only $0.5 \%$. The lower quantity of predicted PE/PPE proteins in MAB was somewhat expected. $M$. tuberculosis has five ESX secretion systems, four of which encode PE/PPE proteins, while MAB has only two (ESX-3 and ESX-4) of which only the ESX-3 operon includes

Table 4 Abundance of Antigenic Regions (AAR) for M. abscessus and M. tuberculosis strains

\begin{tabular}{lll}
\hline Strain & Number of proteins in the dataset & Average AAR value \\
\hline M. tuberculosis Beijing isolate C3 & 37.52 \\
M. tuberculosis Beijing isolate C4 & 37.55 \\
M. bovis BCG Pasteur & 553 & 38.99 \\
M. abscessus ATCC 19977 & 519 & $\mathbf{4 0 . 7 8}$ \\
M. tuberculosis H37Rv & $\mathbf{8 8 6}$ & 40.37 \\
M. abscessus clinical isolates & 548 & 41.54 \\
\hline
\end{tabular}

a Both Beijing isolates were previously reported in Cornejo-Granados et al. [19] 
$\mathrm{PE} / \mathrm{PPE}$ genes [36]. In contrast, Mce domains are known for participating in host cell entry by mycobacteria [37]. Thus, their higher abundance in MAB as compared to MTB highlights the importance of this pathway for MAB survival within the host. It needs to be mentioned though that Kumar et al. [37], also suggested that in low virulence bacteria, transport activities could be the primary function of Mce operons.

To compare the predicted secretomes according to colony morphotype, we first established the core secretome for the $\mathrm{R}$ and $\mathrm{S}$ variants per subspecies, thus eliminating individualities among the different isolates (Fig. $3)$. The high overall agreement between the core secretomes for both morphotypes of approximately $90 \%$ was expected, considering the fact that $\mathrm{R}$ variants can arise from the $\mathrm{S}$ morphotypes during persistent infection by loss of surface-exposed GPLs caused by mutations in the GPL synthesis pathway [26, 38-40]. However, both the higher number and the higher antigenic densities (lower AAR values) of the ES proteins exclusively found in $R$ variants indicate that additional genetic changes may evolve during S-to- $\mathrm{R}$ conversion. Moreover, this observation raises the question whether some strains with additional genetic traits associated with virulence are able to undergo S-to- $\mathrm{R}$ conversion and cause disease due to $\mathrm{R}$ variants more easily than others. Genomic studies involving sequentially isolated $\mathrm{S}$ and $\mathrm{R}$ variants of the same strain obtained from individual patients over time will be required to better characterize the microevolution of MAB strains within the chronically infected host.

Similarly, the fact that MAB causes both chronic pulmonary disease (with $\mathrm{R}$ variants sometimes increasing over time) and extrapulmonary manifestations (mostly caused by $\mathrm{S}$ variants) led us to investigate whether differences exist in the predicted secretomes of isolates related to these clinical presentations. The absence of major differences in the GO, KEGG, and antigenicity analyses suggest that secretome variations do not influence $M A B$ tissue tropism. Consequently, host characteristics such as severe immunosuppression may be the main driver for invasive MAB infections. Likewise, in the case of tissue infections, which often occur following surgical interventions, insufficient hygiene procedures and sterilization protocols for surgical equipment appear to be more relevant than pathobiological traits such as the secretome intrinsic to the causative MAB isolate [41].

Lastly, we observed that the predicted secretomes of all investigated clinical MAB isolates were less antigenic than the secretomes of $M$. tuberculosis $\mathrm{H} 37 \mathrm{Rv}$ and two clinical $M$. tuberculosis isolates. Additionally, although there was no statistical difference, the isolates with a rough phenotype tended to be more antigenic than the isolates with a smooth phenotype. Previous evidence with M. tuberculosis [19] showed that clinical isolates from the Beijing phenotype showed increased virulence and less antigenic secretomes than the reference strain H37Rv. Thus, the diminished antigenicity of MAB could be viewed as a virulence trait in itself as it would support colonization of the host for extended time periods without immediate progression into clinical disease. However, further experimental tests on antigenicity are needed to demonstrate this observation.

This study represents the first systematic prediction and in silico characterization of the MAB secretome. We acknowledge that an important constraint in this study is the limited total number of genomes analyzed per subspecies and biological source. Thus, care must be taken to not over interpret the findings related to sample subcategories such as subspecies and morphotypes. Also, published experimental data on MAB secretomes are very limited and no systematic validation of the in silico findings reported herein could be performed against such datasets. Although more research will be needed to determine experimental secretomes in NTM, our study demonstrates that using bioinformatics strategies can help to broadly explore mycobacterial secretomes including those of clinical isolates and to tailor subsequent, complex and time-consuming experimental approaches accordingly. This approach can support a systematic investigation of mycobacterial secretomes exploring candidate proteins suitable for developing new vaccines and diagnostic markers to distinguish between colonization and infection.

\section{Methods}

\section{Clinical isolates}

We selected $15 \mathrm{MAB}$ clinical isolates comprising members of all MAB subspecies $\left(\mathrm{MAB}_{\mathrm{A}}, n=7 ; \mathrm{MAB}_{\mathrm{B}}, n=4\right.$; $\left.\mathrm{MAB}_{\mathrm{M}}, \mathrm{n}=4\right)$ and both $\mathrm{S}(n=8)$ and $\mathrm{R}(n=6)$ morphotypes (not determined, $n=1$ ). The strains were isolated from different biological sources representing both pulmonary colonization / infection (sputum, $n=10$ ) and extrapulmonary samples (skin, $n=1$; soft tissue, $n=1$; lymph nodes, $n=2$; blood, $n=1$ ) (Table 1 and Additional file 1: Table S1). For routine diagnostic purposes, species identification was performed using GenoType NTM-DR line probe assays (HAIN Lifescience, Nehren, Germany) and sequencing of the $16 \mathrm{~S}$ and $r p o B$ genes as described previously [42].

\section{Whole genome sequencing and genome assemblies} Genomic DNA (gDNA) of the 15 MAB clinical isolates was extracted from solid cultures using a Centrimonium bromide chloroform DNA extraction protocol as previously described [43]. DNA libraries were constructed with the Nextera XT kit from Illumina and sequenced on the Illumina MiSeq benchtop platform with a v3 chemistry paired - end run and a read lenght of $2 \times 300$ 
bp. We processed the resulting reads with Trimmomatic [44], clipping the Illumina adapter sequences and trimming the reads with a sliding window of $20 \mathrm{bp}$ looking for quality $>30$ and discarding all reads shorter than $100 \mathrm{bp}$. Trimmed reads were used to construct de novo assemblies using SPADES [45] with default parameters and the --careful option enabled. Then, each assembly was analyzed with RAST [46] to obtain all the open reading frames (ORFs). Additionally, we predicted the ORFs from the deposited genome sequence of the $M$. abscessus ATCC 19977 type strain (GenBank CU458896.1) (Additional file 1: Table S1).

\section{Secretome prediction}

The complete set of predicted ORFs was independently analyzed for each genome using the bioinformatics pipeline previously reported by Cornejo-Granados et al. [19] and summarized in Additional file 2: Figure S1. Briefly, we used six different feature-based tools (SignalP, SecretomeP, LipoP, TatP, TMHMM and Phobius) [47-51] to identify ES proteins by the different secretion pathways and to remove the ones that had transmembrane domains (Additional file 2: Fig. S1). The proteins assigned as not-secreted (non-ES) were further classified into transmembrane proteins (TM) if they showed the presence of transmembrane domains with TMHMM 2.0 [50], and into intracellular proteins (incell) if they did not contain any transmembrane domains.

\section{Annotation and comparative analysis of secreted proteins} To assign functional annotations to the proteins present in our genomes, we performed a BLASTP query of those proteins against the non-redundant (nr) complete database using Blast2GO [52] with an E-value cut-off set at 1.0E-3. Furthermore, all proteins were associated with protein families through InterProScan [53] and functionally mapped to Gene Ontology (GO) terms by setting the following parameters: E-value-hi-filter: 1.0E-3; Annotation cut-off: 55; GO weight: 5 and Hsp-Hit Coverage cut-off: 0. Blast2GO was then used to identify over- and under-represented GO and Enzyme Commission (EC) numbers in the ES proteins by setting the significance filter $p$-value to $\leq 0.05$. Also, we used the KEGG Automatic Annotation Server (KAAS) database [54] to assign the pathway annotation to the secreted proteins using the $\mathrm{BBH}$ (bidirectional best hit) method and the reference gene data set assigned to Mycobacterium.

To determine differences between the predicted secretomes in relation to MAB subspecies and morphotype, we established core secretomes by performing a bidirectional best-hit BLASTP search (E-value 1.0E-3) between the ES proteins of all genomes belonging to the respective subspecies and morphotypes. Then, we identified the shared and unique proteins for each comparison.
Additionally, we determined the set of homologous ES proteins shared between the MAB reference strain ATCC 19977 and both M. tuberculosis H37Rv predicted and experimental secretomes [19]. The resulting proteins were further investigated for sequence similarities against known drug targets available on the Drug Bank database (http://www.drugbank.ca/), setting the E-value to 1.0E-3 and all other options to default. In Additional file 8: Table S6, we show all proteins that have similarity with an approved drug target, as well as the drugs that can affect said target.

Additionally, we analyzed the presence of the core secretomes in $20 \mathrm{M}$. abscessus genomes per subspecies downloaded from NCBI (Additional file 4: Table S3). To this end, each downloaded genome was analyzed with RAST to obtain all the open reading frames (ORFs). Next, we performed a BLASTP search (E-value 1.0E-3) of each core secretome against each genome of the corresponding subspecies, and all hit proteins were considered homologs.

\section{Calculation of the abundance of antigenic regions}

The Abundance of Antigenic Regions (AAR) value is used to estimate the antigenic density of a protein by calculating the number of antigenic regions and normalizing it to the sequence length [18]. Of note, proteins with higher antigenic densities have lower AAR values. For this study, we calculated the AAR value for each protein in each data set using the Secret-AAR webserver (http://microbiomics.ibt.unam.mx/tools/aar/index. php) and reported the average unless stated otherwise [55]. Then, we used a Mann-Whitney statistical test to establish any significant differences between the AAR values of the different protein data sets.

\section{Abbreviations}

MAB: Mycobacterium abscessus; NTM: Non-tuberculous mycobacterium; MTBC: M. tuberculosis complex; MTB: M. tuberculosis; TB: Tuberculosis; ES: Excreted / secreted; R: Rough; S: Smooth; MABA: M. abscessus subsp. abscessus; $\mathrm{MAB}_{B}$ : M. abscessus subsp. bolletii; $\mathrm{MAB}_{\mathrm{M}}$ : M. abscessus subsp. massiliense; non-ES: Not-secreted; TM: Transmembrane; incell: Intracellular; AAR: Abundance of Antigenic Regions; MLST: Multilocus Sequence Typing; ST: Sequence Type

\section{Supplementary Information}

The online version contains supplementary material available at https://doi. org/10.1186/s12864-021-07670-7.

Additional file 1: Table S1. Complete metadata of the 15 clinical isolates of $M$. abscessus genomes sequenced.

Additional file 2: Figure S1. Bioinformatics pipeline to indentify and analyze the secreted proteins of $M$. abscessus.

Additional file 3: Table S2. Statistic data of the de novo assemblies for the sequenced isolates.

Additional file 4: Table S3. Comparison of the core secretome of each subspecies vs NCBI genomes. 
Additional file 5: Table S4. AAR values for random constructed secretomes of the rough and smooth phenotypes.

Additional file 6: Figure S2. GO enrichment analysis of enzymes for $M$. abscessus ATCC19977. Percentage of sequences annotated with each GO term for the secreted proteins (blue) and the complete proteins in the genome (red).

Additional file 7: Table S5. List of 222 M. abscessus proteins with homologues in M. tuberculosis H37Rv and with previous experimental support for secretion according to Cornejo-Granados et al. [19].

Additional file 8: Table S6. Potential drug targets for 13 proteins shared between M. tuberculosis H37Rv and M. abscessus ATCC19977.

\section{Acknowledgements}

We would like to thank Julia Zallet and Vanessa Mohr for excellent technical assistance and Henrik Nielsen for his assistance with the SecrtomeP software.

\section{Authors' contributions}

Conceived and designed the experiments: FCG, AOL, FPM. Performed the experiments: FCG, JHR. Analyzed the data: FCG, TAK, FVS, SA, JHR, CU, SN, FPM, AOL. Contributed reagents/materials/analysis tools: AOL, RHP. Wrote and edited the manuscript: FCG, FVS, JHR, RHP, AOL, FPM. All authors read and approved the final version of the manuscript.

\section{Funding}

Parts of this work have been supported by the German Center for Infection Research and Grants by Joachim Herz Foundation, Hamburg, and Mukoviszidose Institut $\mathrm{gGmbH}$, Bonn, the research and development arm of the German Cystic Fibrosis Association Mukoviszidose e.V. to F.P.M. We acknowledge the support provided by CONACyT grant CB- 2013-223279 and SALUD-2014-C01-234188 to A.O.L. This research also received support by the DGAPA PAPIIT UNAM (IN215520) to A.O.L. F.C.G. acknowledges the support of CONACYT as a Postgraduate fellow.

\section{Availability of data and materials}

The reference genomes analyzed for M. abscessus ATCC19977 and M. tuberculosis H37Rv were taken from NCBI, under GenBank IDs CU458896.1 and NC_000962.3, respectively. The Whole Genome Shotgun project has been deposited at NCBI, under BioProject PRJNA646278. It can be accessed with the link https://www.ncbi.nlm.nih.gov/bioproject/PRJNA646278. All the predicted secretomes were deposited in the Secret-AAR web-server (http://microbiomics.ibt.unam.mx/tools/aar/index.php).

Additional data supporting the conclusions of this article are included within the article and its additional file(s).

\section{Declarations}

\section{Ethics approval and consent to participate}

Ethical review and approval was not required for the study as all work was performed on bacterial isolates archived at the strain repository of the National Reference Center for Mycobacteria in Borstel, Germany, in accordance with local legislation and institutional requirements. In particular, no data allowing identification of the affected patients was shared or released and no human DNA was sequenced or analyzed.

\section{Consent for publication}

Not applicable.

\section{Competing interests}

The authors declare that they have no competing interests.

\section{Author details}

'Departamento de Microbiología Molecular, Instituto de Biotecnología, Universidad Nacional Autonoma de México, Cuernavaca, Morelos, Mexico. ${ }^{2}$ Molecular and Experimental Mycobacteriology, Research Center Borstel, Borstel, Germany. ${ }^{3}$ German Center for Infection Research (DZIF), Partner site Hamburg-Lübeck-Borstel, Borstel, Germany. ${ }^{4}$ National and WHO Supranational Reference Center for Mycobacteria, Research Center Borstel, Leibniz Lung Center, Borstel, Germany. ${ }^{5}$ Experimental Pathology Section, National Institute of Medical Sciences and Nutrition Salvador Zubirán, Mexico
City, Mexico. ${ }^{6}$ Institute of Medical Microbiology, Virology and Hospital Hygiene, University Medical Center Hamburg-Eppendorf, Hamburg, Germany.

Received: 3 March 2021 Accepted: 27 April 2021

Published online: 25 May 2021

\section{References}

1. Nessar R, Cambau E, Reyrat J-M, Murray A, Gicquel B. Mycobacterium abscessus: a new antibiotic nightmare. J Antimicrob Chemother. 2012;67(4): 810-8. https://doi.org/10.1093/jac/dkr578.

2. Lee M-R, Sheng W-H, Hung C-C, Yu C-J, Lee L-N, Hsueh P-R. Mycobacterium abscessus complex infections in humans. Emerg Infect Dis. 2015;21(9):163846. https://doi.org/10.3201/2109.141634

3. Sanguinetti M, Ardito F, Fiscarelli E, La Sorda M, D'Argenio P, Ricciotti G, et al. Fatal pulmonary infection due to multidrug-resistant Mycobacterium abscessus in a patient with cystic fibrosis. J Clin Microbiol. 2001;39(2):816-9. https://doi.org/10.1128/JCM.39.2.816-819.2001.

4. Jarand J, Levin A, Zhang L, Huitt G, Mitchell JD, Daley CL. Clinical and microbiologic outcomes in patients receiving treatment for Mycobacterium abscessus pulmonary disease. Clin Infect Dis. 2011;52(5):565-71. https://doi. org/10.1093/cid/ciq237.

5. Chen J, Zhao L, Mao Y, Ye M, Guo Q, Zhang Y, et al. Clinical efficacy and adverse effects of antibiotics used to treat Mycobacterium abscessus pulmonary disease. Front Microbiol. 2019;10:1977. https://doi.org/10.3389/ fmicb.2019.01977.

6. Tortoli E, Kohl TA, Brown-Elliott BA, Trovato A, Leão SC, Garcia MJ, et al. Emended description of Mycobacterium abscessus, Mycobacterium abscessus subsp. abscessus and Mycobacteriumabscessus subsp. bolletii and designation of Mycobacteriumabscessus subsp. massiliense comb. nov. Int J Syst Evol Microbiol. 2016;66(11):4471-9. https://doi.org/10.1099/ijsem.0.0013 76.

7. Howard ST, Rhoades E, Recht J, Pang X, Alsup A, Kolter R, et al. Spontaneous reversion of Mycobacterium abscessus from a smooth to a rough morphotype is associated with reduced expression of glycopeptidolipid and reacquisition of an invasive phenotype. Microbiol Microbiol Soc. 2006;152:1581-90.

8. Abeles SR, Pride DT. Molecular bases and role of viruses in the human microbiome. J Mol Biol. 2014;426(23):3892-906. https://doi.org/10.1016/j. jmb.2014.07.002

9. Ripoll F, Deshayes C, Pasek S, Laval F, Beretti J-L, Biet F, et al. Genomics of glycopeptidolipid biosynthesis in Mycobacterium abscessus and $\mathrm{M}$. chelonae. BMC genomics. BioMed Central. 2007;8:114-9.

10. Gutiérrez AV, Viljoen A, Ghigo E, Herrmann J-L, Kremer L. Glycopeptidolipids, a Double-Edged Sword of the Mycobacterium abscessus Complex. Front Microbiol Front. 2018;9:1145

11. Tjalsma H, Antelmann H, Jongbloed JDH, Braun PG, Darmon E, Dorenbos R, et al. Proteomics of protein secretion by Bacillus subtilis: separating the "secrets" of the secretome. Microbiol Mol Biol Rev. 2004;68(2):207-33. https://doi.org/10.1128/MMBR.68.2.207-233.2004.

12. Daugelat S, Guile H, Schoel B, Kaufmann SHE. Secreted antigens of Mycobacterium tuberculosis: characterization with T lymphocytes from patients and contacts after two-dimensional separation. J Infect Dis. 1992; 166(1):186-90. https://doi.org/10.1093/infdis/166.1.186.

13. Zheng J, Ren X, Wei C, Yang J, Hu Y, Liu L, et al. Analysis of the secretome and identification of novel constituents from culture filtrate of bacillus Calmette-Guerin using high-resolution mass spectrometry. Mol Cell Proteomics. 2013;12(8):2081-95. https://doi.org/10.1074/mcp.M113.027318.

14. Gupta MK, Subramanian V, Yadav JS. Immunoproteomic identification of secretory and subcellular protein antigens and functional evaluation of the secretome fraction of Mycobacterium immunogenum, a newly recognized species of the Mycobacterium chelonae-Mycobacterium abscessus group. J Proteome Res. 2009;8(5):2319-30. https://doi.org/10.1021/pr8009462.

15. Shin A-R, Sohn H, Won CJ, Lee B, Kim WS, Kang HB, et al. Characterization and identification of distinct Mycobacterium massiliense extracellular proteins from those of Mycobacterium abscessus. J Microbiol. 2010;48:50211 The Microbiological Society of Korea.

16. Yadav JS, Gupta M. Secretome differences between the taxonomically related but clinically differing mycobacterial species Mycobacterium abscessus and M. chelonae. JIOMICS. 2012;2:1-16.

17. Laencina L, Dubois V, Le Moigne V, Viljoen A, Majlessi L, Pritchard J, et al. Identification of genes required for Mycobacterium abscessus growth 
in vivo with a prominent role of the ESX-4 locus. Proc Natl Acad Sci U.S.A. 2018;115:E1002-11 National Academy of Sciences.

18. Gomez S, Adalid-Peralta L, Palafox-Fonseca H, Cantu-Robles VA, Soberón X, Sciutto $E$, et al. Genome analysis of excretory/secretory proteins in Taenia solium reveals their abundance of antigenic regions (AAR). Sci Rep. 2015; 5(1):9683. https://doi.org/10.1038/srep09683.

19. Cornejo-Granados F, Zatarain-Barrón ZL, Cantu-Robles VA, Mendoza-Vargas A, Molina-Romero C, Sánchez F, et al. Secretome Prediction of Two M. tuberculosis clinical isolates reveals their high antigenic density and potential drug targets. Front Microbiol. 2017;8:128.

20. Macheras E, Konjek J, Roux AL, Thiberge JM, Bastian S, Leão SC, et al. Multilocus sequence typing scheme for the Mycobacterium abscessus complex. Res Microbiol. 2014;165(2):82-90. https://doi.org/10.1016/j.resmic.2013.12.003.

21. Wang S, Wei W, Cai X. Genome-wide analysis of excretory/secretory proteins in Echinococcus multilocularis: insights into functional characteristics of the tapeworm secretome. Parasit Vectors. 2015;8(1):666. https://doi.org/10.1186/s13071-015-1282-7.

22. Tortoli E, Fedrizzi T, Meehan CJ, Trovato A, Grottola A, Giacobazzi E, et al. The new phylogeny of the genus Mycobacterium: the old and the news. Infect Genet Evol. 2017;56:19-25. https:/doi.org/10.1016/j.meegid.2017.10.013.

23. Malhotra S, Vedithi SC, Blundell TL. Decoding the similarities and differences among mycobacterial species. Yang R, editor. PLoS Negl Trop Dis. 2017;11: e0005883 Public Library of Science.

24. Bachmann NL, Salamzade R, Manson AL, Whittington R, Sintchenko V, Earl $\mathrm{AM}$, et al. Key transitions in the evolution of rapid and slow growing Mycobacteria identified by comparative genomics. Front Microbiol Front. 2019;10:3019.

25. Ripoll F, Pasek S, Schenowitz C, Dossat C, Barbe V, Rottman M, et al. Non mycobacterial virulence genes in the genome of the emerging pathogen Mycobacterium abscessus. Ahmed N, editor. PLoS ONE. 2009;4:e5660 Public Library of Science.

26. Ryan K, Byrd TF. Mycobacterium abscessus: shapeshifter of the mycobacterial world. Front Microbiol. 2018;9:2642. https://doi.org/10.3389/ fmicb.2018.02642.

27. Waman VP, Vedithi SC, Thomas SE, Bannerman BP, Munir A, Skwark MJ, et al. Mycobacterial genomics and structural bioinformatics: opportunities and challenges in drug discovery. Emerg Microbes Infect. 2019;8(1):109-18. https://doi.org/10.1080/22221751.2018.1561158.

28. O'Toole N, Min XJ, Butler G, Storms R, Tsang A. Sequence-based analysis of fungal secretomes. Appl Mycol Biotechnol. 2013;6:277-96 Elsevier B.V.

29. Mukherjee S, Bassler BL. Bacterial quorum sensing in complex and dynamically changing environments. Nat Rev Microbiol Nature Publishing Group. 2019;17:371-82.

30. Orme IM, Ordway DJ. Host response to nontuberculous mycobacterial infections of current clinical importance. Andrews-Polymenis $\mathrm{HL}$, editor. Infect Immun. 2014;82:3516-22 American Society for Microbiology Journals.

31. Clary G, Sasindran SJ, Nesbitt N, Mason L, Cole S, Azad A, et al. Mycobacterium abscessus smooth and rough morphotypes form antimicrobial-tolerant biofilm phenotypes but are killed by acetic acid. Antimicrob Agents Chemother. 2018;62:117 American Society for Microbiology Journals.

32. Kulka K, Hatfull G, Ojha AK. Growth of Mycobacterium tuberculosis biofilms. Washington D.C.: JoVE; 2012.

33. Maurer FP, Bruderer VL, Ritter C, Castelberg C, Bloemberg GV, Böttger EC. Lack of antimicrobial bactericidal activity in Mycobacterium abscessus. 2nd ed. Antimicrob Agents Chemothe. 2014;58:3828-36 American Society for Microbiology Journals.

34. Faria S, Joao I, Jordao L. General overview on Nontuberculous mycobacteria, biofilms, and human infection. J Pathog Hindawi. 2015;2015:809014-0.

35. Hunt-Serracin AC, Parks BJ, Boll J, Boutte CC. Mycobacterium abscessus cells have altered antibiotic tolerance and surface glycolipids in artificial cystic fibrosis sputum medium. Antimicrobial Agents Chemother. 2019;63:1370 American Society for Microbiology Journals.

36. Dumas E, Christina Boritsch E, Vandenbogaert M, de la Vega RC R, Thiberge J-M, Caro V, et al. Mycobacterial pan-genome analysis suggests important role of plasmids in the radiation of type VII secretion systems. Genome Biol Evol. 2016;8(2):387-402. https://doi.org/10.1093/gbe/evw001.

37. Kumar A, Chandolia A, Chaudhry U, Brahmachari V, Bose M. Comparison of mammalian cell entry operons of mycobacteria: in silico analysis and expression profiling. FEMS Immunol Med Microbiol. 2005:43(2):185-95. https://doi.org/10.1016/j.femsim.2004.08.013.
38. Catherinot E, Clarissou J, Etienne G, Ripoll F, Emile JF, Daffé M, et al. Hypervirulence of a rough variant of the Mycobacterium abscessus type strain. Infect Immun. 2007;75(2):1055-8. https://doi.org/10.1128/IAl.00835-06.

39. Roux A-L, Viljoen A, Bah A, Simeone R, Bernut A, Laencina L, et al. The distinct fate of smooth and rough Mycobacterium abscessus variants inside macrophages. Open Biol. 2016;6:160185 The Royal Society.

40. Bernut A, Herrmann J-L, Kissa K, Dubremetz J-F, Gaillard J-L, Lutfalla G, et al. Mycobacterium abscessus cording prevents phagocytosis and promotes abscess formation. Proc Natl Acad Sci U.S.A. 2014;111:E943-52 National Academy of Sciences.

41. Maurer F, Castelberg C, Braun von A, Wolfensberger A, Bloemberg G, Bottger $E$, et al. Postsurgical wound infections due to rapidly growing mycobacteria in Swiss medical tourists following cosmetic surgery in Latin America between 2012 and 2014. Euro Surveill. 2014;19:20905 European Centre for Disease Prevention and Control.

42. Adekambi T, Colson P, Drancourt M. rpoB-based identification of nonpigmented and late-pigmenting rapidly growing mycobacteria. J Clin Microbiol. 2003;41:5699-708 American Society for Microbiology Journals.

43. De Almeida IN, Da Silva CW, Rossetti ML, Costa ERD, De Miranda SS. Evaluation of six different DNA extraction methods for detection of Mycobacterium tuberculosis by means of PCR-IS6110: preliminary study. BMC Res Notes. 2013;6(1):561-6. https://doi.org/10.1186/1756-0500-6-561 BioMed Central.

44. Bolger AM, Lohse M, Usadel B. Trimmomatic: a flexible trimmer for Illumina sequence data. Bioinformatics. 2014;30(15):2114-20. https://doi.org/10.1093/ bioinformatics/btu170

45. Bankevich A, Nurk S, Antipov D, Gurevich AA, Dvorkin M, Kilikov AS, et al. SPAdes: a new genome assembly algorithm and its applications to single cell sequencing. J Comput Biol. 2012;19(5):455-77. https://doi.org/10.1089/ cmb.2012.0021.

46. Aziz RK, Bartels D, Best AA, Dejongh M, Disz T, Edwards RA, et al. The RAST server: rapid annotations using subsystems technology. BMC Genomics. 2008;9(1):75. https://doi.org/10.1186/1471-2164-9-75.

47. Petersen TN, Brunak S, Heijne von G, Nielsen H. SignalP 4.0: discriminating signal peptides from transmembrane regions. Nat Methods. 2011;8:785-6 Nature Publishing Group.

48. Bendtsen JD, Kiemer L, Fausbøø A, Brunak S. Non-classical protein secretion in bacteria. BMC Microbiol. 2005;5(1):58. https://doi.org/10.1186/1471-2180-5-58.

49. Bendtsen JD, Nielsen $H$, Widdick D, Palmer T, Brunak S. Prediction of twinarginine signal peptides. BMC Bioinformatics. 2005;6(1):167. https://doi.org/1 $0.1186 / 1471-2105-6-167$

50. Sonnhammer EL, Heijne von G, Krogh A. A hidden Markov model for predicting transmembrane helices in protein sequences. Proc Int Conf Intell Syst Mol Biol. 1998;6:175-82.

51. Käll L, Krogh A, Sonnhammer ELL. Advantages of combined transmembrane topology and signal peptide prediction--the Phobius web server. Nucleic Acids Res. 2007;35(Web Server):W429-32. https://doi.org/10.1093/nar/gkm256.

52. Conesa A, Götz S. Blast2GO: a comprehensive suite for functional analysis in plant genomics. Int J Plant Genomics. 2008;2008:619832.

53. Zdobnov EM, Apweiler R. InterProScan - an integration platform for the signature-recognition methods in InterPro. Bioinformatics. 2001;17(9):847-8. https://doi.org/10.1093/bioinformatics/17.9.847.

54. Moriya Y, Itoh M, Okuda S, Yoshizawa AC, Kanehisa M. KAAS: an automatic genome annotation and pathway reconstruction server. Nucleic Acids Res. 2007;35(Web Server):W182-5. https://doi.org/10.1093/nar/gkm321.

55. Cornejo-Granados F, Hurtado-Ramírez JM, Hernandez-Pando R, Ochoa-Leyva A. Secret-AAR: a web server to assess the antigenic density of proteins and homology search against bacterial and parasite secretome proteins. Genomics. 2019;111(6):1514-6. https://doi.org/10.1016/.ygeno.2018.10.007.

\section{Publisher's Note}

Springer Nature remains neutral with regard to jurisdictional claims in published maps and institutional affiliations. 